\title{
Interaction of a Magnet and a Point Charge: Unrecognized Internal Electromagnetic Momentum
}

\author{
Timothy H. Boyer \\ Department of Physics, City College of the City \\ University of New York, New York, New York 10031
}

\begin{abstract}
Whereas nonrelativistic mechanics always connects the total momentum of a system to the motion of the center of mass, relativistic systems, such as interacting electromagnetic charges, can have internal linear momentum in the absence of motion of the center of energy of the system. This internal linear momentum of the system is related to the controversial concept of "hidden momentum." We suggest that the term "hidden momentum" be abandoned. Here we use the relativistic conservation law for the center of energy to give an unambiguous definition of the "internal momentum of a system," and then we exhibit this internal momentum for the system of a magnet (modeled as a circular ring of moving charges) and a distant static point charge. The calculations provide clear illustrations of this system for three cases: a) the moving charges of the magnet are assumed to continue in their unperturbed motion, b) the moving charges of the magnet are free to accelerate but have no mutual interactions, and c) the moving charges of the magnet are free to accelerate and also interact with each other. It is noted that when the current-carrying charges of the magnet are allowed to interact, the magnet itself will contain internal electromagnetic linear momentum, something which has not been presented clearly in the research and teaching literature.
\end{abstract}




\section{INTRODUCTION}

\section{A. Problem of the Interaction of a Magnet and a Point Charge}

Although the idea of electromagnetic field momentum is more than a century old, there is still confusion regarding this momentum. This confusion can be found in connection with a magnet and a point charge. Now the interaction of a magnet and point charge might seem to be so basic that it should be well-understood in a junior-level course in electromagnetism. However, the actual situation is quite the contrary. The momentum contained in stationary distributions of electric charges and currents is still being debated in teaching and research journals, $\frac{1}{=}$ and the subject is tied up with the controversy over what is termed "hidden

momentum." $\underline{\underline{2}}$ In the present article, we will discuss these questions yet again in the context of an unambiguous definition of the "internal momentum of a system" and will then illustrate the concepts involved with a simple model for a magnet.

\section{B. Outline of the Presentation}

The first step in our presentation is a review of the fourth conservation law involving either Galilean invariance or Lorentz invariance. ${ }^{3}$ (The first three conservation laws involve energy, linear momentum, and angular momentum.) We note that relativistic theories allow the possibility of internal momentum in a system which is unrelated to the motion of the center of energy of the system. We use the fourth conservation law to give an unambiguous definition of a system's internal momentum. We view the clear idea of the "internal momentum of a system" as a replacement for the ambiguous term "hidden momentum" which has provided the basis for an extended controversy: $\frac{2}{}$

The focus of our presentation involves internal momentum in the electromagnetic system consisting of a magnet and a point charge. This is the system where the concept of "hidden momentum" first arose. This simple system illustrates the several basic variations of internal momentum. In addition, this system reveals a form of internal electromagnetic linear momentum which does not seem to be presented clearly in either the research or teaching literature.

We will use a point-charge model for the magnet. Thus our magnet can be pictured as a set of point charges moving on a frictionless circular ring with a point charge of opposite 
sign at the center so as to give neutrality to the magnet. Now it appears that some physicists object to the use of a point-charge model, and apparently insist that classical electromagnetism must be discussed in terms of continuous charge and current distributions. However, today, a century after Lorentz's classical electron theory, a point-charge view seems natural, and such a view sometimes gives clarity to difficult systems.

Next we outline the electromagnetic description provided by the Darwin Lagrangian. $\underline{4}^{\underline{*}}$ If we are interested in relativistic point charge systems when radiation is not the subject of interest, then the natural description of classical electrodynamics is that provided by the Darwin Lagrangian. This badly-neglected approximation is frequently helpful; it has been used in discussions of action and reaction between charge particles,,$\underline{5}$ Faraday induction,,$\underline{6}$ massenergy equivalence, $\frac{7}{-}$ and Lorentz transformations of relativistic energy and momentum. $\frac{8}{-}$ Here it is enormously useful when discussing internal momentum.

In the textbooks of electromagnetism, there are many examples and problems where electric currents move in the presence of electrostatic fields $. \underline{9}, \underline{10}$ A static point charge outside a solenoid or a toroid is a typical such situation. However, usually there is no specification as to the behavior of the charges which are carrying the currents. Are the charges held to constant speed by external forces, or are the charges allowed to accelerate but have no mutual interactions, or are the charges allowed to accelerate and also have mutual interactions? Depending on the specific assumption about the behavior of the current-carrying charges, there will be different amounts of momentum in the system. In our examples in this article, we describe these three different assumptions about the charged particle behavior for the simplest situation which contains the basic physics, the situation of a single current loop in the presence of a static point charge.

We start our illustrative calculations with the case in which the currents of the magnet are held at their unperturbed value by nonelectromagnetic external forces. This is the case which presumably is imagined in the literature, though without any acknowledgement that external forces must be present to hold the charges to their unperturbed motion. In this case, the familiar electromagnetic momentum (due to the electric field of the external charge and the magnetic field of the magnet) is an internal momentum for the system of the magnet and the external point charge. We find that the external forces acting on the moving charges of the magnet indeed provide the power flow corresponding to the relativistic conservation law. 
The next example removes the tangential nonelectromagnetic external forces from the moving charges of the magnet but assumes that the moving magnet charges do not interact with each other. In this case, since there are no external forces providing local power to the magnet-point-charge system, there can be no internal linear momentum for the system of the magnet and the static charge. Since there is electromagnetic momentum present (associated with the electric field of the external charge and the magnetic field of the magnet), there must be an additional momentum in the system. Indeed, we review the calculation for the relativistic mechanical momentum in the magnet. For the system of the magnet alone, this mechanical momentum is an internal momentum; the electric field of the external charge provides the external local power needed in the relativistic conservation law. This internal mechanical momentum in the magnet is the familiar "hidden momentum in magnets" in the literature. 11,12

Finally, we consider the case where there are no nonelectromagnetic tangential forces on the charges of the magnet so that these charges can accelerate, but now the charges can also interact. For ease of calculation here, our example involves only two interaction charges. Since we are averaging over the initial orientation of the charges, this two-particle situation is equivalent to many charges when each charge is allowed to interact only with one partner charge on the opposite side of the circular current loop. (A more sophisticated $N$-particle calculation, where there are mutual interactions among all $N$ charges, is carried out in another manuscript. 13 ) Once again, there is no internal momentum for the system of the magnet and the point charge, but there must be internal momentum in the magnet. Here we find that the internal momentum in the magnet is partially mechanical and partially electromagnetic. In the multiparticle case when all the moving charges interact with all the other charges, the internal momentum is overwhelmingly electromagnetic, so that the internal mechanical momentum in the multiparticle magnet is negligible. A brief discussion ends the article.

\section{THE RELATIVISTIC CONSERVATION LAW AND INTERNAL MOMEN- TUM IN A SYSTEM}

The notoriously difficult system of a magnet and a point charge is where Shockley and James 11 introduced the notion of "hidden momentum." Here we wish to discuss this same 
system but to use the idea of internal momentum.

Within nonrelativistic mechanics, all linear momentum is connected to particle mass, $\mathbf{p}=m \mathbf{v}$. The (time derivative of the) conservation law associated with invariance under Galilean transformations is

$$
\mathbf{P}=\left(\sum_{i} m_{i}\right) \mathbf{V}_{c o f m}
$$

where $\mathbf{V}_{\text {cofm }}=d \mathbf{X}_{\text {cof } m} / d t$ and $\mathbf{P}$ is the total system momentum. Thus in nonrelativistic mechanics, the total momentum of the system equals the total mass times the velocity of the center of mass.

In contrast to nonrelativistic mechanics where all momentum is connected to particle mass, electromagnetism contains linear momentum in the electromagnetic fields, $\mathbf{P}_{\text {em }}=$ $[1 /(4 \pi c)] \int d^{3} r \mathbf{E} \times \mathbf{B}$. Since the magnetic field $\mathbf{B}$ is of order $1 / c$, the electromagnetic momentum $\mathbf{P}_{e m}$ is of order $1 / c^{2}$ and corresponds to a relativistic effect. $\underline{\underline{14}}$ The (time derivative of the) conservation law associated with Lorentz transformations takes the form ${ }^{3}$

$$
\sum_{i}\left(\mathbf{F}_{\mathrm{ext} i} \cdot \mathbf{v}_{i}\right) \mathbf{r}_{i}=\frac{d}{d t}\left(U \mathbf{X}_{c \mathrm{of} E}\right)-c^{2} \mathbf{P}
$$

where $\mathbf{F}_{\text {ext } i}$ is the external force applied to the $i$ th particle of the system. This conservation law is sharply different from Eq. (11) associated with Galilean invariance in nonrelativistic mechanics. If we divide the relativistic equation (2) by $c^{2}$ and take $c^{2} \rightarrow \infty$, we indeed recover the nonrelativistic equation (1). However, for a relativistic system, there is a new aspect; the introduction of power $\mathbf{F}_{\text {ext } i} \cdot \mathbf{v}_{i}$ by an external force $\mathbf{F}_{\text {ext } i}$ acting at position $\mathbf{r}_{i}$ leads to a changing position for the center of energy. This situation has no counterpart in the nonrelativistic theory where the introduction of power by an external force has no influence on the center of mass apart from the motion of the masses.

For an isolated relativistic system where no external forces are present, the system $U$ is constant and the relativistic conservation law (2) takes the form

$$
\mathbf{P}=\left(U / c^{2}\right) \mathbf{V}_{c o f}
$$

so that the total momentum of the system is directly related to the velocity of the center of energy. However, the relativistic conservation law (2) now allows a new possibility. Now we can have system momentum which is unrelated to the motion of the center of energy, but rather is related to the local power introduced by external forces. In particular, one can 
find situations where the total energy $U$ and the center of energy $\mathbf{X}_{c o f} E$ are not changing in time, $d\left(U \mathbf{X}_{c o f E}\right) / d t=0$, but there is net linear momentum $\mathbf{P}_{\text {int }}$ given by

$$
\mathbf{P}_{\text {int }}=\frac{-1}{c^{2}} \sum_{i}\left(\mathbf{F}_{\mathrm{ext} i} \cdot \mathbf{v}_{i}\right) \mathbf{r}_{i}
$$

Here there is internal momentum in the system which is related to the local delivery of power by the external forces. This last situation corresponds to that where the presence of "hidden momentum" has been claimed (by some authors) to be present in the system. We notice from the factor of $1 / c^{2}$ in Eq. (4) that we always expect the internal momentum of a system to be of at least order $1 / c^{2}$. The term "hidden momentum," was introduced by Shockley and James 11 in connection with the interaction of a magnet and point charge. However, the term "hidden momentum" seems unfortunate, since it suggests something mysterious.

Furthermore, the use and misuse of the designation has now made the term ambiguous. ${ }^{2}$ Here we will not speak of "hidden momentum" but will refer to the "internal momentum in a system" $\mathbf{P}_{\text {int }}$ if there is linear momentum in the system which is distinct from the motion of the system center of energy. With this definition, a completely isolated system where no external forces are present can contain no internal momentum. On the other hand, a subsystem of some larger system can contain internal momentum in the subsystem due to the forces arising from the other parts of the system; these forces from other parts of the total system are external forces so far as the subsystem is concerned.

\section{BASIS FOR THE CALCULATIONS}

\section{A. The Darwin Lagrangian}

We wish to examine the interaction of a magnet and a point charge through relativistic order $v^{2} / c^{2}$ where electromagnetic field momentum appears. Within classical electrodynamics, the electromagnetic interaction of point charges $e_{i}$ at locations $\mathbf{r}_{i}$ moving with velocity $\mathbf{v}_{i}$ can be described through order $v^{2} / c^{2}$ by the Darwin Lagrangian $\underline{4}^{4}$

$$
\begin{aligned}
\mathcal{L} & =\sum_{i=1}^{i=N} m_{i} c^{2}\left(-1+\frac{\mathbf{v}_{i}^{2}}{2 c^{2}}+\frac{\left(\mathbf{v}_{i}^{2}\right)^{2}}{8 c^{4}}\right)-\frac{1}{2} \sum_{i=1}^{i=N} \sum_{j \neq i} \frac{e_{i} e_{j}}{\left|\mathbf{r}_{i}-\mathbf{r}_{j}\right|} \\
& +\frac{1}{2} \sum_{i=1}^{i=N} \sum_{j \neq i} \frac{e_{i} e_{j}}{2 c^{2}}\left[\frac{\mathbf{v}_{i} \cdot \mathbf{v}_{j}}{\left|\mathbf{r}_{i}-\mathbf{r}_{j}\right|}+\frac{\mathbf{v}_{i} \cdot\left(\mathbf{r}_{i}-\mathbf{r}_{j}\right) \mathbf{v}_{j} \cdot\left(\mathbf{r}_{i}-\mathbf{r}_{j}\right)}{\left|\mathbf{r}_{i}-\mathbf{r}_{j}\right|^{3}}\right] .
\end{aligned}
$$




\section{B. Electric and Magnetic Fields from the Darwin Lagrangian}

The Lagrangian equations of motion following from Eq. (5) can be rewritten in the form of Newton's second law for the mechanical momentum $\mathbf{p}^{\text {mech }}$ and force $\mathbf{F}, d \mathbf{p}^{\text {mech }} / d t=$ $d(m \gamma \mathbf{v}) / d t=\mathbf{F}$, with $\gamma=\left(1-v^{2} / c^{2}\right)^{-1 / 2}$. In this Newtonian form, we have

$$
\begin{aligned}
& \frac{d}{d t}\left[m_{i} \gamma_{i} \mathbf{v}_{i}\right]=\frac{d}{d t}\left[\frac{m_{i} \mathbf{v}_{i}}{\left(1-\mathbf{v}_{i}^{2} / c^{2}\right)^{1 / 2}}\right] \approx \frac{d}{d t}\left[m_{i}\left(1+\frac{\mathbf{v}_{i}^{2}}{2 c^{2}}\right) \mathbf{v}_{i}\right] \\
& =e_{i} \sum_{j \neq i} \mathbf{E}_{j}\left(\mathbf{r}_{i}, t\right)+e_{i} \frac{\mathbf{v}_{i}}{c} \times \sum_{j \neq i} \mathbf{B}_{j}\left(\mathbf{r}_{i}, t\right)
\end{aligned}
$$

with the Lorentz force on the $i$ th particle arising from the electromagnetic fields of the other particles. The electromagnetic fields due to the $j$ th particle are given through order $v^{2} / c^{2}$ by $\underline{\underline{5}}$

$$
\begin{aligned}
\mathbf{E}_{j}(\mathbf{r}, \mathbf{t}) & =e_{j} \frac{\left(\mathbf{r}-\mathbf{r}_{j}\right)}{\left|\mathbf{r}-\mathbf{r}_{j}\right|^{3}}\left[1+\frac{\mathbf{v}_{j}^{2}}{2 c^{2}}-\frac{3}{2}\left(\frac{\mathbf{v}_{j} \cdot\left(\mathbf{r}-\mathbf{r}_{j}\right)}{c\left|\mathbf{r}-\mathbf{r}_{j}\right|}\right)^{2}\right] \\
& -\frac{e_{j}}{2 c^{2}}\left(\frac{\mathbf{a}_{j}}{\left|\mathbf{r}-\mathbf{r}_{j}\right|}+\frac{\mathbf{a}_{j} \cdot\left(\mathbf{r}-\mathbf{r}_{j}\right)\left(\mathbf{r}-\mathbf{r}_{j}\right)}{\left|\mathbf{r}-\mathbf{r}_{j}\right|^{3}}\right)
\end{aligned}
$$

and

$$
\mathbf{B}_{j}(\mathbf{r}, t)=e_{j} \frac{\mathbf{v}_{j}}{c} \times \frac{\left(\mathbf{r}-\mathbf{r}_{j}\right)}{\left|\mathbf{r}-\mathbf{r}_{j}\right|^{3}}
$$

where in Eq. (7) the quantity $\mathbf{a}_{j}$ refers to the acceleration of the $j$ th particle.

\section{Canonical Linear Momentum from the Darwin Lagrangian}

The canonical linear momentum $\mathbf{p}_{i}=\mathbf{p}_{i}^{\text {mech }}+\mathbf{p}_{i}^{\text {field }}$ associated with the $i$ th charge is given by

$$
\frac{\partial \mathcal{L}}{\partial \mathbf{v}_{i}}=\mathbf{p}_{i}=m_{i}\left(1+\frac{\mathbf{v}_{i}^{2}}{2 c^{2}}\right) \mathbf{v}_{i}+\sum_{j \neq i} \frac{e_{i} e_{j}}{2 c^{2}}\left(\frac{\mathbf{v}_{j}}{\left|\mathbf{r}_{i}-\mathbf{r}_{j}\right|}+\frac{\mathbf{v}_{j} \cdot\left(\mathbf{r}_{i}-\mathbf{r}_{j}\right)\left(\mathbf{r}_{i}-\mathbf{r}_{j}\right)}{\left|\mathbf{r}_{i}-\mathbf{r}_{j}\right|^{3}}\right),
$$

corresponding to the mechanical linear momentum $\mathbf{p}_{i}^{\text {mech }}$ of the $i$ th particle

$$
\mathbf{p}_{i}^{\text {mech }}=m_{i} \gamma_{i} \mathbf{v}_{i} \approx m_{i}\left[1+v_{i}^{2} /\left(2 c^{2}\right)\right] \mathbf{v}_{i}=m_{i} \mathbf{v}_{i}+m_{i} v_{i}^{2} \mathbf{v}_{i} /\left(2 c^{2}\right),
$$

and the electromagnetic linear momenta $\mathbf{p}_{i}^{\text {field }}$ associated with the electric field of the $i$ th particle and magnetic fields of all the other $j$ th particles $\frac{15}{15}$

$$
\mathbf{p}_{i}^{f i e l d}=\sum_{j \neq i} 1 /(4 \pi c) \int d^{3} r \mathbf{E}_{i} \times \mathbf{B}_{j}=\sum_{j \neq i} \frac{e_{i} e_{j}}{2 c^{2}}\left(\frac{\mathbf{v}_{j}}{\left|\mathbf{r}_{i}-\mathbf{r}_{j}\right|}+\frac{\mathbf{v}_{j} \cdot\left(\mathbf{r}_{i}-\mathbf{r}_{j}\right)\left(\mathbf{r}_{i}-\mathbf{r}_{j}\right)}{\left|\mathbf{r}_{i}-\mathbf{r}_{j}\right|^{3}}\right) .
$$


Thus the total linear momentum $\mathbf{P}$ is given by

$$
\mathbf{P}=\sum_{i} \mathbf{p}_{i}=\sum_{i} m_{i}\left(1+\frac{\mathbf{v}_{i}^{2}}{2 c^{2}}\right) \mathbf{v}_{i}+\frac{1}{2} \sum_{i} \sum_{j \neq i} \frac{e_{i} e_{j}}{2 c^{2}}\left(\frac{\mathbf{v}_{j}}{\left|\mathbf{r}_{i}-\mathbf{r}_{j}\right|}+\frac{\mathbf{v}_{j} \cdot\left(\mathbf{r}_{i}-\mathbf{r}_{j}\right)\left(\mathbf{r}_{i}-\mathbf{r}_{j}\right)}{\left|\mathbf{r}_{i}-\mathbf{r}_{j}\right|^{3}}\right) .
$$

The Darwin Lagrangian satisfies the familiar conservation laws involving energy, linear momentum, and angular momentum. Through order $v^{2} / c^{2}$ the Darwin Lagrangian satisfies the fourth (and only specifically relativistic) conservation law for relativistic systems involving the uniform motion of the center of energy. Thus the Darwin Lagrangian allows us to discuss systems which contain internal linear momentum in the sense of Eqs. (21) and (4).

\section{BASIC MODEL FOR THE MAGNET-POINT-CHARGE INTERACTION}

\section{A. Magnet Modeled by Moving Point Charges}

The model for a magnet used in our calculations involves $N$ particles $e_{i}$, each of charge $e$ and mass $m$, which are held by external centripetal forces of constraint in a circular orbit of radius $R$ centered on the origin in the $x y$-plane while an opposite neutralizing charge $Q=$ $-N e$ is located at the origin. The charges $e_{i}$ in the circular orbit are free to move along the orbit and will accelerate due to any forces which are tangential to the orbit. Thus in essence, the magnet is pictured as charged beads $e$ sliding on a frictionless ring in the $x y$-plane with a balancing opposite charge $Q$ located at the center of the ring.

In the absence of any perturbing influence, the charges $e_{i}$ can be equally spaced around the circular orbit with phases $\phi_{0 i}=\omega_{0} t+\theta_{i}$ where $\theta_{i}$ is an initial phase. The charges move with angular velocity $\omega_{0}$, speed $v_{0}=\omega_{0} R$, displacement

$$
\mathbf{r}_{0 i}=R\left[\widehat{x} \cos \left(\omega_{0} t+\theta_{i}\right)+\widehat{y} \sin \left(\omega_{0} t+\theta_{i}\right)\right]=\widehat{r}_{0 i} R
$$

and velocity

$$
\mathbf{v}_{0 i}=\omega_{0} R\left[-\widehat{x} \sin \left(\omega_{0} t+\theta_{i}\right)+\widehat{y} \cos \left(\omega_{0} t+\theta_{i}\right)\right]=\widehat{\phi}_{0 i} \omega_{0} R
$$

Here the radial and tangential unit vectors for the charge $e_{i}$ are

$$
\widehat{r}_{0 i}=\widehat{x} \cos \left(\omega_{0} t+\theta_{i}\right)+\widehat{y} \sin \left(\omega_{0} t+\theta_{i}\right)
$$

and

$$
\widehat{\phi}_{0 i}=-\widehat{x} \sin \left(\omega_{0} t+\theta_{i}\right)+\widehat{y} \cos \left(\omega_{0} t+\theta_{i}\right)
$$


The magnetic moment $\vec{\mu}$ of the $N$-moving-particle magnet is given by the time-average of $e \mathbf{r} \times \mathbf{v} /(2 c)$ corresponding to

$$
\vec{\mu}=\left\langle\sum_{i=1}^{N} e \mathbf{r}_{i} \times \mathbf{v}_{i} /(2 c)\right\rangle=\widehat{z} N e R^{2} \omega_{0} /(2 c) .
$$

We now introduce an external charge $q$ located on the $x$-axis at coordinate $x_{q}, \mathbf{r}_{q}=\widehat{x} x_{q}$, which is held in place by nonelectromagnetic external forces of constraint. If the charge $q$ is far away from the magnet, $R<<x_{q}$, then the electric field $\mathbf{E}_{q}(\mathbf{r})$ near the position of the magnet, $r \approx R<<x_{q}$, is given by

$$
\mathbf{E}_{q}(\mathbf{r})=\frac{q\left(\mathbf{r}-\mathbf{r}_{q}\right)}{\left|\mathbf{r}-\mathbf{r}_{q}\right|^{3}} \approx \frac{q\left(\mathbf{r}-\widehat{x} x_{q}\right)}{x_{q}^{3}}\left(1+\frac{3 \widehat{x} \cdot \mathbf{r}}{x_{q}}\right)=\frac{-\widehat{x} q}{x_{q}^{2}}+\frac{q[\mathbf{r}-3 \widehat{x}(\widehat{x} \cdot \mathbf{r})]}{x_{q}^{3}} \approx \frac{-\widehat{x} q}{x_{q}^{2}}
$$

to order $R / x_{q}$. In this article, we will need only the leading term in the electric field, $\mathbf{E}_{q}(0) \approx-\widehat{x} q / x_{q}^{2}$.

We notice that for our model, the magnetic moment $\vec{\mu}$ in Eq. (17) will remain unchanged provided that the average speed $\left(\sum_{i} v_{i}\right) / N$ of the the magnet charges remains unchanged. Since the magnet charges move in a circular orbit in the $x y$-plane, $\mathbf{r}_{i} \times \mathbf{v}_{i}=\widehat{z} R v_{i}$, and so the only possible change in the magnetic moment is associated with a change in the average speed of the magnet charges. In the calculations to follow, the average speed is unchanged by the presence of the static charge $q$ and so the magnetic moment is unchanged.

The central negative charge $Q$ will make no contribution to the momentum of the magnet in our subsequent calculations since this charge is at rest at the center of the circular ring. The canonical momentum $\mathbf{p}_{Q}$ of the central charge $Q$ has no mechanical contribution because the charge is at rest, and the electromagnetic field contribution vanishes because the moving magnet charges have zero average velocity, which is unchanged by any perturbation due to the external static charge $q$. Thus from Eq. (9), we have

$$
\mathbf{p}_{Q}=M_{Q} 0+\sum_{i=1}^{N} \frac{Q e}{2 c^{2}}\left(\frac{\mathbf{v}_{i}}{R}+\frac{\mathbf{v}_{i} \cdot\left(\mathbf{r}_{i}-0\right)\left(\mathbf{r}_{i}-0\right)}{R^{3}}\right)=0
$$

since $\mathbf{v}_{i} \cdot \mathbf{r}_{i}=0$ and in all our calculations $\sum_{i} \mathbf{v}_{i}=0$. 


\section{MAGNET-POINT-CHARGE INTERACTION FOR UNPERTURBED MAG- NET CURRENTS}

\section{A. Familiar Electromagnetic Field Momentum for an Unperturbed Current Loop in an External Electric Field}

In the approximation that the charges $e_{i}$ of the magnet are not perturbed by the presence of the point charge $q$, we can obtain the linear momentum in the electromagnetic field as the contribution of the electric field of the point charge $q$ and the magnetic field arising from the moving magnet charges $e_{i}$. This corresponds to the canonical momentum of the stationary charge $q$ (which is entirely electromagnetic field momentum),

$$
\begin{aligned}
\mathbf{p}_{q} & =\mathbf{p}_{q}^{\text {field }}=\sum_{i=1}^{N} \frac{1}{4 \pi c} \int d^{3} r \mathbf{E}_{q} \times \mathbf{B}_{i}=\sum_{j=1}^{N} \frac{q e}{2 c^{2}}\left(\frac{\mathbf{v}_{j}}{\left|\mathbf{r}_{q}-\mathbf{r}_{j}\right|}+\frac{\mathbf{v}_{j} \cdot\left(\mathbf{r}_{q}-\mathbf{r}_{j}\right)\left(\mathbf{r}_{q}-\mathbf{r}_{j}\right)}{\left|\mathbf{r}_{q}-\mathbf{r}_{j}\right|^{3}}\right) \\
& \approx \sum_{j=1}^{N} \frac{q e}{2 c^{2}}\left[\frac{\mathbf{v}_{j}}{x_{q}}\left(1+\frac{\widehat{x} \cdot \mathbf{r}_{j}}{x_{q}}\right)+\frac{\mathbf{v}_{j} \cdot\left(\widehat{x} x_{q}-\mathbf{r}_{j}\right)\left(\widehat{x} x_{q}-\mathbf{r}_{j}\right)}{x_{q}^{3}}\left(1+\frac{3 \widehat{x} \cdot \mathbf{r}_{j}}{x_{q}}\right)\right]
\end{aligned}
$$

We now insert the expressions (13) and (14) into Eq. (20), average over time, and keep

terms through first order $1 / x_{q}^{2}$. We note that $\mathbf{v}_{j} \cdot \mathbf{r}_{j}=0,\left\langle\mathbf{v}_{j}\right\rangle=0,\left\langle\left(\mathbf{v}_{j} \cdot \widehat{x}\right)\left(\widehat{x} \cdot \mathbf{r}_{j}\right)\right\rangle=0$, and $\left\langle\mathbf{v}_{j}\left(\widehat{x} \cdot \mathbf{r}_{j}\right)\right\rangle=\widehat{y} \omega_{0} R^{2} / 2=-\left\langle\left(\mathbf{v}_{j} \cdot \widehat{x}\right) \mathbf{r}_{j}\right\rangle$. The time-averaged electromagnetic field momentum $\mathbf{p}_{q}$ receives equal contributions from each charge, giving

$$
\left\langle\mathbf{p}_{q}\right\rangle=\frac{\widehat{y} N q e \omega_{0} R^{2}}{2 c^{2} x_{q}^{2}}=\frac{1}{c} \mathbf{E}_{q}(0) \times \vec{\mu}
$$

This is the familiar expression for the electromagnetic field momentum for an unperturbed localized steady current in an external electric field; it corresponds to a problem in Jackson's text. $\underline{10}$ Even if the currents of the magnet are perturbed by the electric field $\mathbf{E}_{q}(0)$, the field momentum in Eq. (21) will remain unchanged to lowest order in this perturbing field.

\section{B. Relativistic Conservation Law and Internal Momentum for Unperturbed Cur-} rents in the Magnet

In most treatments of the interaction a magnet and a point charge, it is not pointed out that (in the presence of the external charge $q$ ) the constant currents of the magnet are made possible by nonelectromagnetic tangential forces $\mathbf{F}_{\text {exti }}$ on the charges $e_{i}$ carrying the magnet's currents. These nonelectromagnetic external forces $\mathbf{F}_{\text {ext } i}$ on the charges $e_{i}$ balance 
the tangential force $e \mathbf{E}_{q}\left(\mathbf{r}_{i}\right)$ of the electric field due to the external charge $q$. Since these charges $e_{i}$ are moving in the electric field of the charge $q$, the electric fields deliver local power $e \mathbf{E}_{q}\left(\mathbf{r}_{i}\right) \cdot \mathbf{v}_{i}$ which must be absorbed by the nonelectromagnetic forces $\mathbf{F}_{\text {ext } i}$ which keep the charges $e_{i}$ moving with constant speed. Then the quantity on the left-hand side of the relativistic conservation law in Eq. (2) and on the right-hand side of Eq. (41) corresponds to

$$
\begin{aligned}
\frac{1}{c^{2}} \sum_{i}\left(\mathbf{F}_{\text {ext } i} \cdot \mathbf{v}_{i}\right) \mathbf{r}_{i} & =\frac{1}{c^{2}} \sum_{i}\left(-e \mathbf{E}_{q}\left(\mathbf{r}_{i}\right) \cdot \mathbf{v}_{i}\right) \mathbf{r}_{i}=\frac{1}{c^{2}} \sum_{i}\left(e \frac{\widehat{x}}{x_{q}^{2}} \cdot \mathbf{v}_{i}\right) \mathbf{r}_{i} \\
& =-\frac{\widehat{y} N q e \omega_{0} R^{2}}{2 c^{2} x_{q}^{2}}=-\left\langle\mathbf{p}_{q}\right\rangle
\end{aligned}
$$

where we have again used $\widehat{y} \omega_{0} R^{2} / 2=-\left\langle\left(\mathbf{v}_{j} \cdot \widehat{x}\right) \mathbf{r}_{j}\right\rangle$. Thus exactly as expected, the internal momentum of the system of the point charge and the magnet with unperturbed currents is non-zero, and this internal momentum corresponds exactly to the electromagnetic field momentum involving the electric field of the charge $q$ and the magnetic field of the magnet given in Eq. (21). This familiar electromagnetic momentum is not usually classified as "hidden momentum" in the muddled literature of "hidden momentum." However, this momentum is exactly linear momentum which does not involve motion of the center of energy of the system and is associated with the introduction of local power as in the form (4) of the relativistic conservation law.

In this case, there is no internal momentum in the subsystem of the magnet alone; the equally-spaced magnet particles move with constant speed in a circle so $\sum_{i} \mathbf{p}_{i}=0$. This fits with the relativistic Eq. (4) since there is no net local delivery of power by external forces on the magnet; the electric forces $e \mathbf{E}_{q}\left(\mathbf{r}_{i}\right)$ (which are now external forces) on the magnetic charges $e_{i}$ due to the charge $q$ are balanced by the nonelectromagnetic tangential forces $\mathbf{F}_{\text {ext } i}$, so that the power delivered by the electric field of the charge $q$ is balanced by the power absorbed by the nonelectromagnetic tangential forces. 


\section{MAGNET-POINT-CHARGE INTERACTION FOR FREELY-MOVING NON- INTERACTING CHARGES IN THE MAGNET}

\section{A. Perturbation of the One-Moving-Particle Magnet}

Our next model for the magnet currents involves $N$ non-interacting charges which are free to move in the direction tangential to the circular orbit. Thus we may picture the charges as sliding freely on the frictionless ring in response to the electric field of the external charge. Since the charges have no mutual interactions, we may treat each charge separately as though the magnet involved only one moving charge of mass $m$ and charge $e$. For this one-movingparticle magnet, the index " $i$ " corresponds only to $i=1$. The neutralizing charge $Q=-e$ is located at the origin.

The position of the charged particle $e$ in the magnet is perturbed by the presence of the external electric field due to the charge $q$. The phase angle is no longer the $\phi_{0 i}=\omega_{0} t+\theta_{i}$ appearing in Eq. (13) , but rather becomes $\phi_{i}=\omega_{0} t+\theta_{i}+\eta_{i}(t)$, so that the displacement of the particle through first order in the perturbation is now

$$
\mathbf{r}_{i}=R\left\{\widehat{x} \cos \left[\omega_{0} t+\theta_{i}+\eta_{i}(t)\right]+\widehat{y} \sin \left[\omega_{0} t+\theta_{i}+\eta_{i}(t)\right]\right\}=R \widehat{r}_{i}=\mathbf{r}_{0 i}+\delta \mathbf{r}_{i}
$$

where

$$
\widehat{r}_{i}=\widehat{x} \cos \left[\omega_{0} t+\theta_{i}+\eta_{i}(t)\right]+\widehat{y} \sin \left[\omega_{0} t+\theta_{i}+\eta_{i}(t)\right],
$$

the unperturbed displacement $\mathbf{r}_{0 i}$ is given in Eq. (13), and

$$
\delta \mathbf{r}_{i}=\eta_{i} R\left\{-\widehat{x} \sin \left[\omega_{0} t+\theta_{i}\right]+\widehat{y} \cos \left[\omega_{0} t+\theta_{i}\right]\right\}=\widehat{\phi}_{0 i} R \eta_{i}
$$

Due to the perturbation, the velocity $\mathbf{v}_{i}=d \mathbf{r}_{i} / d t$ is now

$$
\begin{aligned}
\mathbf{v}_{i} & =\left(\omega_{0}+d \eta_{i} / d t\right) R\left\{-\widehat{x} \sin \left[\omega_{0} t+\theta_{i}+\eta_{i}(t)\right]+\widehat{y} \cos \left[\omega_{0} t+\theta_{i}+\eta_{i}(t)\right]\right\} \\
& =\widehat{\phi}_{i}\left(\omega_{0}+d \eta_{i} / d t\right) R=\mathbf{v}_{0 i}+\delta \mathbf{v}_{i}
\end{aligned}
$$

where

$$
\widehat{\phi}_{i}=-\widehat{x} \sin \left[\omega_{0} t+\theta_{i}+\eta_{i}(t)\right]+\widehat{y} \cos \left[\omega_{0} t+\theta_{i}+\eta_{i}(t)\right],
$$

the unperturbed velocity $\mathbf{v}_{0 i}$ is given in Eq. (14), and

$$
\delta \mathbf{v}_{i}=\widehat{\phi}_{0 i} R d \eta_{i} / d t-\widehat{r}_{0 i} R \omega_{0} \eta_{i}
$$


through first order in the perturbation $\eta_{i}$. In obtaining Eqs. (25) and (28), we have used Eqs. (15) and (16) as well as the small-angle approximations $\cos (\phi+\delta \phi) \approx \cos \phi-\delta \phi \sin \phi$ and $\sin (\phi+\delta \phi) \approx \sin \phi+\delta \phi \cos \phi$

The mechanical momentum in Eq. (10) involves two terms, $\mathbf{p}_{i}^{\text {mech }}=m_{i} \mathbf{v}_{i}+m_{i} v_{i}^{2} \mathbf{v}_{i} /\left(2 c^{2}\right)$. When averaged in time, the first term vanishes, $\left\langle m_{i} \mathbf{v}_{i}\right\rangle=m_{i}\left\langle\mathbf{v}_{i}\right\rangle=0$, since for a stationary situation the time-average velocity vanishes. Consequently, the average mechanical momentum in a stationary situation involves terms which already contain a factor of $1 / c^{2}$, $\left\langle\mathbf{p}_{i}^{\text {mech }}\right\rangle=\left\langle m_{i} v_{i}^{2} \mathbf{v}_{i} /\left(2 c^{2}\right)\right\rangle$, so that the velocity $\mathbf{v}_{i}$ needs to be calculated only through nonrelativistic order.

\section{B. Calculation of the Perturbation by Energy Conservation}

For the one-moving-particle magnet (or $N$-non-interacting-moving-particle magnet), it is convenient to obtain the perturbed phase $\eta_{i}(t)$ from energy conservation. The centripetal forces of constraint do no work, and hence the total energy (kinetic plus electrostatic particle energy) of the particle (or of each particle of a non-interacting group) is conserved,

$$
\frac{1}{2} m v_{0 i}^{2}=\frac{1}{2} m v_{i}^{2}+\left(e q / x_{q}^{2}\right) R \cos \left[\omega_{0} t+\theta_{i}+\eta_{i}(t)\right]
$$

where the last term is the potential energy $-e_{i} \mathbf{E}_{q}(0) \cdot \mathbf{r}_{i}$ of the magnet charge $e$ in the approximately uniform electrostatic field of the external charge $q$. Now we are interested in the behavior of the system through first order in the perturbation $e q / x_{q}^{2}$. Thus we expand

each of the terms on the right-hand side of Eq. (29). Expanding the particle kinetic energy $m v^{2} / 2$, we have from Eqs. (14), (26) and (28)

$$
\frac{1}{2} m v_{i}^{2}=\frac{1}{2} m\left(\mathbf{v}_{0 i}+\delta \mathbf{v}\right)^{2}=\frac{1}{2} m v_{0 i}^{2}+m \mathbf{v}_{0 i} \cdot \delta \mathbf{v}_{i}=\frac{1}{2} m v_{0 i}^{2}+m \omega_{0} R^{2} \frac{d \eta_{i}}{d t} .
$$

Also, the term involving the cosine in Eq. (29) is already first order in the perturbation, and therefore we may drop the $\eta_{i}$ in the argument of the cosine. The energy conservation equation then becomes

$$
\frac{1}{2} m v_{0 i}^{2}=\frac{1}{2} m v_{0 i}^{2}+m \omega_{0} R^{2} \frac{d \eta_{i}}{d t}+\frac{e q}{x_{q}^{2}} R \cos \left(\omega_{0} t+\theta_{i}\right)
$$

or

$$
\frac{d \eta_{i}}{d t}=-\frac{e q}{x_{q}^{2} m \omega_{0} R} \cos \left(\omega_{0} t+\theta_{i}\right) .
$$


Integrating once, we have the perturbing phase as

$$
\eta_{i}(t)=-\frac{e q}{x_{q}^{2} m \omega_{0}^{2} R} \sin \left(\omega_{0} t+\theta_{i}\right) .
$$

\section{Mechanical Momentum of the Perturbed One-Moving-Particle Magnet}

Since the external charge $q$ is not moving, the canonical momentum $\mathbf{p}_{e}$ given in Eq. (9) for the one-moving-particle magnet consists entirely of mechanical momentum. We can now calculate the average mechanical linear momentum of the charge $e$ which is moving in a circular orbit. Through first order in the perturbing force $e q / x_{q}^{2}$ and second order in $v_{0} / c$, the mechanical momentum $\mathbf{p}_{m}^{\text {mech }}$ is given from Eqs. (10) and (26) by

$$
\begin{aligned}
\mathbf{p}_{e} & =\mathbf{p}_{m}^{\text {mech }}=m \gamma_{e} \mathbf{v}_{e}=m\left[1+v_{0}^{2} /\left(2 c^{2}\right)+\mathbf{v}_{0} \cdot \delta \mathbf{v}_{i} / c^{2}\right]\left(\mathbf{v}_{0 i}+\delta \mathbf{v}_{i}\right) \\
& =m\left(\mathbf{v}_{0 i}+\delta \mathbf{v}_{i}\right)+\left[v_{0}^{2} /\left(2 c^{2}\right)\right]\left(\mathbf{v}_{0 i}+\delta \mathbf{v}_{i}\right)+m\left(\mathbf{v}_{0} \cdot \delta \mathbf{v}_{i}\right) \mathbf{v}_{0 i} / c^{2}
\end{aligned}
$$

Then averaging in time and noting that $\left\langle\mathbf{v}_{0 i}\right\rangle=\left\langle\delta \mathbf{v}_{i}\right\rangle=0$, we have from Eq. (14), (28), (33) and (파),

$$
\begin{aligned}
\mathbf{p}_{e} & =\left\langle\frac{m\left(\mathbf{v}_{0} \cdot \delta \mathbf{v}_{i}\right) \mathbf{v}_{0 i}}{c^{2}}\right\rangle=\left\langle\frac{m \omega_{0} R^{2}}{c^{2}} \frac{d \eta_{i}}{d t} \mathbf{v}_{0 i}\right\rangle \\
& =\left\langle\frac{m \omega_{0} R^{2}}{c^{2}}\left(-\frac{e q}{x_{q}^{2} m \omega_{0} R} \cos \left(\omega_{0} t+\theta_{i}\right)\right) \omega_{0} R\left[-\widehat{x} \sin \left(\omega_{0} t+\theta_{i}\right)+\widehat{y} \cos \left(\omega_{0} t+\theta_{i}\right)\right]\right\rangle \\
& =-\widehat{y} \frac{e q \omega_{0} R^{2}}{2 c^{2} x_{q}^{2}} .
\end{aligned}
$$

This result is just the negative of Eq. (21) when $N=1$. Thus the average mechanical momentum (for the single charge $e$ of the one-moving-particle magnet in the presence of the charge $q$ ) is equal in magnitude and opposite in sign from the canonical momentum $\mathbf{p}_{q}$ corresponding to the familiar electromagnetic field momentum associated with the electric field of the external charge $q$ and the magnetic field of the magnet. Thus the total momentum of the system consisting of the charge $q$ and the one-moving-particle magnet indeed vanishes,

$$
\langle\mathbf{P}\rangle=\left\langle\mathbf{p}_{q}\right\rangle+\left\langle\mathbf{p}_{e}\right\rangle=\left\langle\mathbf{p}_{q}^{\text {field }}\right\rangle+\left\langle\mathbf{p}_{e}^{\text {mech }}\right\rangle=\widehat{y} \frac{e q \omega_{0} R^{2}}{2 x_{q}^{2} c^{2}}-\widehat{y} \frac{e q \omega_{0} R^{2}}{2 x_{q}^{2} c^{2}}=0,
$$

as required by the relativistic conservation law. In this case, there are no nonelectromagnetic forces which introduce local power into the system of the magnet and the point charge $q$, and hence the total linear momentum of this system must vanish as required by Eq. (3). 
On the other hand, if we consider the magnet alone as our system, then the electric forces $e \mathbf{E}_{q}\left(\mathbf{r}_{i}\right)$ of the external charge $q$ are now external forces on the magnet, and these external forces provide local power $e \mathbf{E}_{q}\left(\mathbf{r}_{i}\right) \cdot \mathbf{v}_{i}$ to charges of the magnet. Thus as far as the magnet alone is concerned, the power delivery by the external forces is

$$
\frac{1}{c^{2}} \sum_{i}\left(e \mathbf{E}_{q}\left(\mathbf{r}_{i}\right) \cdot \mathbf{v}_{i}\right) \mathbf{r}_{i}=\frac{1}{c^{2}} \sum_{i}\left(e \frac{-\widehat{x}}{x_{q}^{2}} \cdot \mathbf{v}_{i}\right) \mathbf{r}_{i}=\frac{\widehat{y} N q e \omega_{0} R^{2}}{2 c^{2} x_{q}^{2}}=-\left\langle\mathbf{p}_{e}\right\rangle,
$$

which agrees exactly with the mechanical momentum in the magnet, as required by the relativistic conservation law in the form (44). The linear momentum $\left\langle\mathbf{p}_{e}\right\rangle$ given in Eq. (35) is what is identified in the literature as the "hidden mechanical momentum" in the magnet.

\section{INTERNAL ELECTROMAGNETIC MOMENTUM IN THE TWO- INTERACTING-MOVING-PARTICLE MAGNET}

\section{A. Improved Model of Two Moving Magnet Charges which Interact}

The physical magnets found in nature do not consist of a single charged particle (or of non-interacting particles) sliding on a frictionless ring. Hence we turn to a model consisting of particles which interact pair-wise with a partner on the opposite side of the ring as an improvement over our noninteracting-particle model for a magnet. Since each pair of particles does not interact with any other pair, we may treat the magnet model as involving two interacting moving charged particles. The limit to pairwise interactions is made here simply for ease of calculation; a fully-interacting calculation is carried out elsewhere. $\underline{13}$ Thus now we have two charges $e$ held by external centripetal forces of constraint in a circular orbit of radius $R$ centered on the origin in the $x y$-plane while a neutralizing charge $Q=-2 e$ is located at the origin. The calculation for the magnetic moment $\vec{\mu}$ and the field momentum associated with the canonical momentum $\mathbf{p}_{q}$ follow as in the calculations above with the results in Eqs. (17) and (21) corresponding to $N=2$.

\section{B. Internal Electromagnetic Field Momentum}

The total linear momentum $\mathbf{P}$ of our example involves not only the electromagnetic field momentum $\mathbf{p}_{q}=\mathbf{p}_{q}^{\text {field }}$ associated with the canonical momentum of the stationary particle 
$q$ but also the canonical momenta $\mathbf{p}_{e 1}$ and $\mathbf{p}_{e 2}$ associated with the particles of the magnet. Since the charge $q$ is at rest, it has no magnetic field, and hence it does not contribute to the canonical momentum of $\mathbf{p}_{e 1}$ or $\mathbf{p}_{e 2}$. However, the canonical momentum $\mathbf{p}_{e 1}$ of the first magnet particle includes both its mechanical momentum and also the electromagnetic momentum associated with its own electric field and the magnetic field of the other moving particle in the magnet. Now the unperturbed motion of the magnet charges given in Eqs. (13) and (14) involves no average linear momentum because the two charges are always moving with opposite velocities on opposite sides of the circular orbit. However, the perturbed motion will indeed involve net linear momentum for the magnet particles. As soon as the particles of the magnet have mutual interactions, the mechanical kinetic energy changes (which provided the basis for the internal mechanical momentum in the one-movingparticle magnet) are suppressed as energy goes into electrostatic energy of the interacting particles. The internal mechanical momentum of the magnet decreases because of the electrostatic interactions, and internal electromagnetic momentum appears in the internal electromagnetic fields. We will illustrate this situation explicitly for our example involving the two-interacting-moving-particle magnet.

\section{Calculation of the Perturbation Using Nonrelativistic Forces}

In order to obtain the internal linear momentum $\mathbf{p}_{e 1}+\mathbf{p}_{e 2}$ of the magnet in the presence of the external electric field $\mathbf{E}_{q}$ due to the charge $q$, we need to calculate the perturbed motion of the particles $e_{1}$ and $e_{2}$. The perturbed positions of the two charges $e_{1}$ and $e_{2}$ of the magnet will be written as in Eq. (23), where the unperturbed initial phases differ by $\pi, \phi_{01}-\phi_{02}=\theta_{1}-\theta_{2}=\pi$, and where it is again assumed that $\eta_{i}(t)$ is a small correction. The perturbed velocities of the charges are as given in Eq. (26) , and the accelerations then follow as

$$
\begin{aligned}
\mathbf{a}_{i} & =\left(\omega_{0}+d \eta_{i} / d t\right)^{2} R\left\{-\widehat{x} \cos \left[\omega_{0} t+\theta_{i}+\eta_{i}(t)\right]-\widehat{y} \sin \left[\omega_{0} t+\theta_{i}+\eta_{i}(t)\right]\right\} \\
& +\left(d^{2} \eta_{i} / d t^{2}\right) R\left\{-\widehat{x} \sin \left[\omega_{0} t+\theta_{i}+\eta_{i}(t)\right]+\widehat{y} \cos \left[\omega_{0} t+\theta_{i}+\eta_{i}(t)\right]\right\} \\
& =-\widehat{r}_{i}\left(\omega_{0}+d \eta_{i} / d t\right)^{2} R+\widehat{\phi}_{i}\left(d^{2} \eta_{i} / d t^{2}\right) R
\end{aligned}
$$

where $\widehat{r}_{i}$ and $\widehat{\phi}_{i}$ are given in Eqs. (24) and (27).

Since the magnet charges $e_{1}$ and $e_{2}$ are constrained to move in a circular orbit, the 
perturbation of the charges is determined by the tangential acceleration. For charge $e_{i}$, the equation of motion requires only the electrostatic forces due to the stationary charge $q$ and the moving charge $e_{j \neq i}$. There are relativistic fields of order $1 / c^{2}$ in the tangential direction due to the moving charge $e_{i \neq j}$, but these $1 / c^{2}$-corrections will not contribute to average momentum of the magnet because the expression (11) for the electromagnetic momentum of the magnet is itself of order $1 / c^{2}$. The radial forces are balanced by the forces of constraint. Thus from Eq. (38), we have for the nonrelativistic equation of motion

$$
\begin{aligned}
m \mathbf{a}_{i} \cdot \widehat{\phi}_{i} & \approx m R \frac{d^{2} \eta_{i}}{d t^{2}} \\
& =\widehat{\phi}_{i} \cdot\left[e \mathbf{E}_{q}\left(\mathbf{r}_{i}, t\right)+e \mathbf{E}_{j \neq i}\left(\mathbf{r}_{i}, t\right)\right]=\widehat{\phi}_{i} \cdot\left(\frac{-\widehat{x} e q}{x_{q}^{2}}+\frac{e^{2}\left(\mathbf{r}_{i}-\mathbf{r}_{j \neq i}\right)}{\left|\mathbf{r}_{i}-\mathbf{r}_{j \neq i}\right|^{3}}\right) \\
& =\left(-\widehat{\phi}_{i} \cdot \widehat{x} \frac{e q}{x_{q}^{2}}-\widehat{\phi}_{i} \cdot \mathbf{r}_{j \neq i} \frac{e^{2}}{\left|\mathbf{r}_{i}-\mathbf{r}_{j \neq i}\right|^{3}}\right)
\end{aligned}
$$

since $\widehat{\phi}_{i} \cdot \mathbf{r}_{i}=0$, where the tangential unit vector is given in Eq. (27) while $\mathbf{r}_{j \neq i}=\widehat{x} R \cos \left[\omega_{0} t+\right.$ $\left.\theta_{i}+\pi+\eta_{j \neq i}(t)\right]+\widehat{y} R \sin \left[\omega_{0} t+\theta_{i}+\pi+\eta_{j \neq i}(t)\right]$. Then we have

$$
\begin{aligned}
\widehat{\phi}_{i} \cdot \mathbf{r}_{j \neq i} & =R\left\{-\sin \left(\omega_{0} t+\theta_{i}+\eta_{i}\right) \cos \left[\omega_{0} t+\theta_{i}+\pi+\eta_{j \neq i}(t)\right]\right. \\
& \left.+\cos \left(\omega_{0} t+\theta_{i}+\eta_{i}\right) \sin \left[\omega_{0} t+\theta_{i}+\pi+\eta_{j \neq i}(t)\right]\right\} \\
& =R \sin \left(\eta_{j \neq i}-\eta_{i}+\pi\right)=-R \sin \left(\eta_{j \neq i}-\eta_{i}\right) \approx R\left(\eta_{i}-\eta_{j \neq i}\right)
\end{aligned}
$$

where we have used the approximation $\sin \phi \approx \phi$ for small $\phi$. The separation $\left|\mathbf{r}_{i}-\mathbf{r}_{j \neq i}\right|$ between the charges is second order in the perturbation $\eta$, and so we may write $\left|\mathbf{r}_{i}-\mathbf{r}_{j \neq i}\right| \approx$ $2 R$ in Eq. (39). Then the nonrelativistic equation of motion (39) for the charge $e_{i}$ through first order in the perturbation produced by $e q / x_{q}^{2}$ becomes

$$
m R \frac{d^{2} \eta_{i}}{d t^{2}}=\left(\frac{e q}{x_{q}^{2}} \sin \left(\omega_{0} t+\theta_{i}\right)-\frac{e^{2}}{(2 R)^{3}} R\left(\eta_{i}-\eta_{j \neq i}\right)\right) .
$$

We notice that since $\phi_{0 i}-\phi_{0 j \neq i}=\theta_{i}-\theta_{j \neq i}=\pi$, the right-hand side of this equation (41) is odd under the interchange of the two particles. Thus for the steady-state situation, we must have

$$
\eta_{j \neq i}=-\eta_{i}
$$

Then the perturbation in the phase $\eta_{i}$ (in steady state) is given by

$$
m R \frac{d^{2} \eta_{i}}{d t^{2}}=\left(\frac{e q}{x_{q}^{2}} \sin \left(\omega_{0} t+\theta_{i}\right)-\frac{e^{2}}{(2 R)^{2}} \eta_{i}\right)
$$


with a steady-state solution

$$
\eta_{i}(t)=\frac{e q}{x_{q}^{2}} \frac{\sin \left(\omega_{0} t+\theta_{i}\right)}{\left[-m \omega_{0}^{2} R+e^{2} /(2 R)^{2}\right]} .
$$

If we take the magnitude $e$ of the charges as small (so that we may neglect the terms in $e^{2}$ involving interactions between the charges), $m \omega_{0}^{2} R \gg e^{2} /(2 R)^{2}$, then equation (44) agrees exactly with the one-particle-magnet result in Eq. (33). Thus we recover the noninteracting particle result in the appropriate small-charge-e limit. On the other hand, if the magnitude $e$ of the charges becomes large, $e^{2} /(2 R)^{2} \gg m \omega_{0}^{2} R$, then according to Eq. (44) the electrostatic interaction contribution can dominate the mechanical contribution. Although both the small-e and large-e results are accurate, the near-resonant situation $-m \omega_{0}^{2} R+e^{2} /(2 R)^{2} \approx 0$ is not allowed by the approximation used in the calculation, that the perturbation $\eta_{i}(t)$ is small, $\left|\eta_{i}(t)\right| \ll 1$.

\section{Internal Momentum in the Magnet}

The internal canonical momentum of the magnet in the presence of the external charge $q$ is given by the sum over the canonical momenta $\mathbf{p}_{i}$ in Eq. (9) where the sum includes only the two charges of the magnet in our model, each with canonical momentum

$$
\mathbf{p}_{e i}=\mathbf{p}_{e i}^{\text {mech }}+\mathbf{p}_{e i}^{\text {field }}=m\left(1+\frac{\mathbf{v}_{i}^{2}}{2 c^{2}}\right) \mathbf{v}_{i}+\frac{e^{2}}{2 c^{2}}\left(\frac{\mathbf{v}_{j \neq i}}{\left|\mathbf{r}_{i}-\mathbf{r}_{j \neq i}\right|}+\frac{\mathbf{v}_{j \neq i} \cdot\left(\mathbf{r}_{i}-\mathbf{r}_{j \neq i}\right)\left(\mathbf{r}_{i}-\mathbf{r}_{j \neq i}\right)}{\left|\mathbf{r}_{i}-\mathbf{r}_{j \neq i}\right|^{3}}\right) .
$$

When averaged in time, we expect equal momentum contributions from each charge. The velocity $\mathbf{v}_{i}$ is given in Eq. (26) where $\eta_{i}$ is given in Eq. (44) and its time derivative is

$$
\frac{d \eta_{i}}{d t}=\omega_{0} \frac{e q}{x_{q}^{2}} \frac{\cos \left(\omega_{0} t+\theta_{i}\right)}{\left[-m \omega_{0}^{2} R+e^{2} /(2 R)^{2}\right]} .
$$

\section{Mechanical Linear Momentum of a Perturbed Magnet Charge}

Then the mechanical contribution $\mathbf{p}_{i}^{\text {mech }}$ to the linear momentum is

$$
\begin{aligned}
\mathbf{p}_{e i}^{\text {mech }} & =m\left(1+\frac{v_{i}^{2}}{2 c^{2}}\right) \mathbf{v}_{i}=m \mathbf{v}_{i}+m \frac{\left(\mathbf{v}_{0 i}+\delta \mathbf{v}_{i}\right)^{2}}{2 c^{2}} \mathbf{v}_{i} \approx m \mathbf{v}_{i}+m \frac{\mathbf{v}_{0 i}^{2}}{2 c^{2}} \mathbf{v}_{i}+m \frac{\mathbf{v}_{0 i} \cdot \delta \mathbf{v}_{i}}{c^{2}} \mathbf{v}_{0 i} \\
& =m \mathbf{v}_{i}+m \frac{\mathbf{v}_{0 i}^{2}}{2 c^{2}} \mathbf{v}_{i}+\frac{m}{c^{2}} \omega_{0} \frac{d \eta_{i}}{d t} R^{2} \mathbf{v}_{0 i}
\end{aligned}
$$


from Eqs. (26) and (28). The average value of the velocity is zero, $\left\langle\mathbf{v}_{i}\right\rangle=0$, since the magnet-charge interaction is assumed stationary. The required average for $\mathbf{p}_{i}^{\text {mech }}$ follows from Eqs. (14), (46), and (47) as

$$
\left\langle\mathbf{p}_{e i}^{\text {mech }}\right\rangle=\left\langle\frac{m}{c^{2}} \omega_{0} \frac{d \eta_{i}}{d t} R^{2} \mathbf{v}_{0 i}\right\rangle=\widehat{y} \frac{m}{c^{2}} \omega_{0}^{2} R^{2} \frac{e q}{x_{q}^{2}} \frac{\omega_{0} R / 2}{\left[-m \omega_{0}^{2} R+e^{2} /(2 R)^{2}\right]} .
$$

\section{Electromagnetic Linear Momentum Associated with a Perturbed Magnet Charge}

The electromagnetic contribution $\mathbf{p}_{i}^{\text {field }}$ corresponds to

$$
\begin{aligned}
\mathbf{p}_{e i}^{f i e l d} & =\frac{e^{2}}{2 c^{2}}\left(\frac{\mathbf{v}_{j \neq i}}{\left|\mathbf{r}_{i}-\mathbf{r}_{j \neq i}\right|}+\frac{\mathbf{v}_{j \neq i} \cdot\left(\mathbf{r}_{i}-\mathbf{r}_{j \neq i}\right)\left(\mathbf{r}_{i}-\mathbf{r}_{j \neq i}\right)}{\left|\mathbf{r}_{i}-\mathbf{r}_{j \neq i}\right|^{3}}\right) \\
& =\frac{e^{2}}{2 c^{2}}\left(\frac{\mathbf{v}_{j \neq i}}{\left|\mathbf{r}_{i}-\mathbf{r}_{j \neq i}\right|}+\frac{\mathbf{v}_{j \neq i} \cdot \mathbf{r}_{i}\left(\mathbf{r}_{i}-\mathbf{r}_{j \neq i}\right)}{\left|\mathbf{r}_{i}-\mathbf{r}_{j \neq i}\right|^{3}}\right)
\end{aligned}
$$

since $\mathbf{v}_{j \neq i} \cdot \mathbf{r}_{j \neq i}=0$. The denominator will involve a distance $2 R$ through first order in the perturbation. We need first

$$
\mathbf{v}_{j \neq i} \cdot \mathbf{r}_{i}=v_{j \neq i} \widehat{\phi}_{j \neq i} \cdot \mathbf{r}_{i} \approx \omega_{0} R^{2}\left(-2 \eta_{i}\right)
$$

from Eqs. (40) and (42). Then from Eqs. (15), (44), and (150), the time-average of $\left(\mathbf{v}_{j \neq i} \cdot \mathbf{r}_{i}\right) \mathbf{r}_{i}$ becomes

$$
\left\langle\left(\mathbf{v}_{j \neq i} \cdot \mathbf{r}_{i}\right) \mathbf{r}_{i}\right\rangle=\left\langle\omega_{0} R^{2}\left(-2 \eta_{i}\right) \mathbf{r}_{i 0}\right\rangle=\left(-2 \omega_{0} R^{2}\right) \frac{e q}{x_{q}^{2}} \frac{1}{\left[-m \omega_{0}^{2} R+e^{2} /(2 R)^{2}\right]} \widehat{y} \frac{R}{2},
$$

and similarly

$$
\left\langle\left(\mathbf{v}_{j \neq i} \cdot \mathbf{r}_{i}\right) \mathbf{r}_{j \neq i}\right\rangle=\left(2 \omega_{0} R^{2}\right) \frac{e q}{x_{q}^{2}} \frac{1}{\left[-m \omega_{0}^{2} R+e^{2} /(2 R)^{2}\right]} \widehat{y} \frac{R}{2} .
$$

Then from Eq. (49), the time-average electromagnetic contribution to $\mathbf{p}_{e i}$ is

$$
\begin{aligned}
\left\langle\mathbf{p}_{e i}^{\text {field }}\right\rangle & =\frac{e^{2}}{2 c^{2}}\left[\left(-2 \omega_{0} R^{2}\right) \frac{e q}{x_{q}^{2}} \frac{2}{\left[-m \omega_{0}^{2} R+e^{2} /(2 R)^{2}\right]} \widehat{y} \frac{R}{2}\left(\frac{1}{(2 R)^{3}}\right)\right] \\
& =-\widehat{y}\left(\frac{e^{2}}{(2 R)^{2}}\right) \frac{\omega_{0} R^{2}}{2 c^{2}} \frac{e q / x_{q}^{2}}{\left[-m \omega_{0}^{2} R+e^{2} /(2 R)^{2}\right]} .
\end{aligned}
$$

Adding the mechanical contribution in Eq. (48) and the electromagnetic contribution in Eq. (53), we find

$$
\begin{aligned}
\left\langle\mathbf{p}_{e i}\right\rangle & =\left\langle\mathbf{p}_{e i}^{\text {mech }}\right\rangle+\left\langle\mathbf{p}_{e i}^{\text {field }}\right\rangle=\widehat{y} \frac{m}{c^{2}} \omega_{0}^{2} R^{2} \frac{e q}{x_{q}^{2}} \frac{\omega_{0} R / 2}{\left[-m \omega_{0}^{2} R+e^{2} /(2 R)^{2}\right]} \\
& -\widehat{y}\left(\frac{e^{2}}{(2 R)^{2}}\right) \frac{\omega_{0} R^{2}}{2 c^{2}} \frac{e q / x_{q}^{2}}{\left[-m \omega_{0}^{2} R+e^{2} /(2 R)^{2}\right]} \\
& =-\frac{\widehat{y} q e \omega_{0} R^{2}}{2 c^{2} x_{q}^{2}}
\end{aligned}
$$


The two equal contributions $\left\langle\mathbf{p}_{e i}\right\rangle$ and $\left\langle\mathbf{p}_{e j \neq i}\right\rangle$ from the two particles give the canonical momentum $\left\langle\mathbf{p}_{\text {magnet }}\right\rangle$ of the magnet as

$$
\left\langle\mathbf{p}_{\text {magnet }}\right\rangle=\left\langle\mathbf{p}_{e i}\right\rangle+\left\langle\mathbf{p}_{e j \neq i}\right\rangle=-2 \frac{\widehat{y} q e \omega_{0} R^{2}}{2 c^{2} x_{q}^{2}} .
$$

But then the canonical momentum $\left\langle\mathbf{p}_{\text {magnet }}\right\rangle$ of the two-particle magnet is equal in magnitude and opposite in sign compared to the canonical momentum $\left\langle\mathbf{p}_{q}\right\rangle$ of the external charge $q$ corresponding to $N=2$ in Eq. (21), which was equal to the familiar electromagnetic field momentum involving the electric field due to $q$ and the magnetic field of the magnet. We see that the relativistic conservation law (2) regarding the center of energy is indeed satisfied, and that the total internal momentum of the system of the magnet and the point charge indeed vanishes.

\section{DISCUSSION OF INTERNAL ELECTROMAGNETIC MOMENTUM}

The magnitudes of $\left\langle\mathbf{p}_{e i}^{\text {mech }}\right\rangle$ in Eq. (48) and of $\left\langle\mathbf{p}_{e i}^{\text {field }}\right\rangle$ in Eq. (153) are in the ratio $m \omega_{0}^{2} R /\left[e^{2} /(2 R)^{2}\right]$. Thus in the limit of small value for the charge $e$ of the magnet particles, the mechanical momentum in the magnet dominates the internal electromagnetic momentum of the magnet. This mechanical momentum corresponds to the "hidden mechanical momentum" of the textbooks and literature. However, in the opposite limit of large charge $e$ for the magnet particles, the internal electromagnetic momentum of the magnet becomes large and the mechanical momentum becomes negligible. As more particles of fixed mass $m$ and charge $e$ are added to the magnet while keeping the magnetic moment $\vec{\mu}$ fixed, the average speed $v_{0 i}=\omega_{0} R$ of the current carriers becomes ever smaller so that the mechanical internal momentum becomes insignificant compared to the internal electromagnetic momentum of the magnet. Thus for any physical multiparticle magnet with its enormous number of charge carriers, we expect that only the internal electromagnetic momentum of the magnet needs to be considered. This internal electromagnetic momentum in the magnet is equal in magnitude and opposite in direction to the electromagnetic field momentum which is found in the elementary textbook calculations involving a point charge and a steady current. The negligible contribution of the particle mechanical momentum to the magnet's internal momentum is analogous to the negligible contribution of the particle kinetic energy to the magnet field energy related to the self-inductance of a circuit ${ }^{6}$ where the mass and the 
charge of the charge carriers is never mentioned in the textbooks. The familiar electromagnetic momentum involving the electric field of the external charge and the magnetic field of the magnet appears in all the textbooks. However, there does not seem to be any discussion of the internal electromagnetic momentum between the electric and magnetic fields of the magnet particles undergoing perturbed motion due to the electric field of the external point charge.

Coleman and Van Vleck ${ }^{12}$ insisted that the "hidden momentum" in magnets of Shockley and James ${ }^{11}$ was purely mechanical. Other authors (such as Furry ${ }^{16}$ ) have not emphasized this aspect. The idea of internal momentum in magnets now appears in the electromagnetism textbooks. Jackson has a problem 17 in his graduate text which considers a point charge at the center of a toroidal magnet. There are no external forces on the system, the center of energy is not moving, and therefore the total linear momentum of the system must vanish. The text correctly suggests that some internal momentum is present in the magnet, but follows Coleman and Van Vleck in referring to this momentum as "mechanical." Also, in his undergraduate text, Griffiths has an example ${ }^{18}$ of a rectangular current loop in an external electric field, and notes correctly that relativistic internal momentum (hidden momentum) is present in the current loop. For noninteracting charges, the internal momentum is indeed " purely mechanical;" however, for interacting charges, the situation would involve internal electromagnetic momentum in the current loop.

\section{ACKNOWLEDGEMENT}

I wish to thank Professor David J. Griffiths for his many helpful comments and suggestions which improved the clarity and accuracy of the present article.

1 D. J. Griffiths, "Resource Letter EM-1: Electromagnetic Momentum," Am. J. Phys. 80, 7-18 (2012).

2 Ref. 1, contains a section listing twenty-three articles on hidden momentum.

3 T. H. Boyer, "Illustrations of the relativistic conservation law for the center of energy," Am. J. Phys. 73, 953-961 (2005); see Eq. (14). See also, T. H. Boyer, "Illustrating some implications 
of the conservation laws in relativistic mechanics," Am. J. Phys. 77, 562-569 (2009); "Examples and comments related to relativity controversies," Am. J. Phys. 80, 962-971 (2012).

4 C. G. Darwin, "The Dynamical Motions of Charged Particles," Phil. Mag. 39, 537-551 (1920). The Darwin Lagrangian appears in a number of advanced textbooks. See, for example, J. D. Jackson, Classical Electrodynamics 3rd edition (Wiley, New York, 1999), pp. 596-598, or J. Schwinger, L. L. DeRaad, Jr., K. A. Milton, and W. Tsai, Classical Electrodynamics (Perseus Books, Reading, MA, 1998), p. 365, or L. D. Landau and E. M. Lifshitz, "The Classical Theory of Fields 4th edition," (Pergamon, New York, 1975), pp. 165-168.

5 L. Page and N. I. Adams, "Action and reaction between moving charges," Am. J. Phys. 13, 141-147 (1945).

6 T. H. Boyer, "Faraday Induction and the Current Carriers in a Circuit," submitted for publication. See arXiv 1410.1197.

7 T. H. Boyer, "Example of mass-energy relation: Classical hydrogen atom accelerated or supported in a gravitational field,"; Am. J. Phys. 66, 872-876 (1998).

8 T. H. Boyer, "Lorentz-transformation properties of energy and momentum in electromagnetic systems," Am. J. Phys. 53, 167-171 (1985).

9 See, for example, D. J. Griffiths, Introduction to Electrodynamics 4th edition (Pearson, New York, 2013), Problem 8.15 on p. 379, Problem 8.20 on p. 380, and Problem 8.22 on p. 381.

10 See, for example, J. D. Jackson in Ref. 4, Problems 6.5 and 6.6 on p. 286.

11 W. Shockley and R. P. James, “Try simplest cases' discovery of 'hidden momentum' forces on 'magnetic currents,"' Phys. Rev. Lett. 18, 876-879 (1967).

12 S. Coleman and J. H. Van Vleck, "Origin of 'hidden momentum forces' on magnets," Phys. Rev. 171, 1370-1375 (1968).

13 See, T. H. Boyer, "Classical Interaction of a Magnet and a Point Charge: The Classical Electromagnetic Forces Responsible for the Aharonov-Bohm Phase Shift," arXiv 1408.3745. The final sections of this manuscript are still undergoing revision.

14 We will use gaussian units throughout our discussion. When treating relativistic aspects of electromagnetic theory, gaussian units are far more natural than S.I.

15 The calculation of $1 /(4 \pi c) \int d^{3} r \mathbf{E}_{i} \times \mathbf{B}_{j}$ for two point charges is essentially the same as the calculation of the vector potential in the Coulomb gauge given on page 597 of Jackson's text in Ref. 4. 
16 W. H. Furry, "Examples of momentum distributions in the electromagnetic field and in matter," Am . J. Phys. 37, 621-636 (1969).

17 See Jackson's text in Ref. 4, Problem 6.6 on pp. 286-287 and Problem 12.8 on p. 618-619.

18 See Griffiths' text in Ref. 9, Example 12.13 on pp. 547-549. 